\title{
1 Passive Assay of Plutonium Metal Plates using a Fast-Neutron 2 Multiplicity Counter
}

\author{
3 A. Di Fulvio ${ }^{1}$, T. H. Shin ${ }^{1}$, T. Jordan ${ }^{1}$, C. Sosa ${ }^{1}$, M. L. Ruch ${ }^{1}$, S.D. Clarke $^{1}$, D. L. Chichester ${ }^{2}$, S. A. Pozzi ${ }^{1}$ \\ $4{ }^{1}$ Department of Nuclear Engineering \& Radiological Sciences, University of Michigan, Ann Arbor MI 48109 U.S.A. \\ $5 \quad{ }^{2}$ Idaho National Laboratory, Idaho Falls, ID 83415 U.S.A.
}

\begin{abstract}
6 Abstract
7 We developed a fast-neutron multiplicity counter based on organic scintillators (EJ-309 liquid and 8 stilbene). The system detects correlated photon and neutron multiplets emitted by fission reactions, 9 within a gate time of tens of nanoseconds. The system was used at Idaho National Laboratory to assay a 10 variety of plutonium metal plates. A coincidence counting strategy was used to quantify the ${ }^{240} \mathrm{Pu}$ 11 effective mass of the samples. Coincident neutrons, detected within a 40-ns coincidence window, show 12 a monotonic trend, increasing with the ${ }^{240}$ Pu-effective mass (in this work, we tested the 0.005-0.5 kg 13 range). After calibration, the system estimated the ${ }^{240} \mathrm{Pu}$ effective mass of an unknown sample $\left({ }^{240} \mathrm{Pu}_{\text {eff }}\right.$ $>50 \mathrm{~g}$ ) with an uncertainty lower than $1 \%$ in a 4 -minute assay time.
\end{abstract}

\section{Introduction and motivation}

Nuclear materials accounting for safeguards applications often requires the non-destructive assay (NDA) of plutonium-bearing materials, such as plutonium metal samples, metallic or oxide fuel rods, cans of plutonium-oxide or mixed-oxide (containing both uranium and plutonium) powder, or scrap/waste materials. Passive neutron coincidence counting is the technique of choice for this application, relying on thermal neutron well counters [1]. A thermal-neutron well counter consists of a ring of ${ }^{3} \mathrm{He}$-based proportional counters, embedded in a cadmium-lined polyethylene assembly. ${ }^{3} \mathrm{He}$-based well counters are nearly insensitive to gamma-rays and have a relatively high intrinsic neutron detection efficiency $(10-30 \%)[2,3]$. However, the coincidence gate is constrained by the relatively long neutron die-away time $(10-300 \mu \mathrm{s})$ for these moderated detector assemblies [4], which is related to the neutron lifetime in the system. Because of this long time-gate, the rate of accidental coincidences due to source and random background events is rather large [5]. Thus, the main practical limitation of thermal systems is the long measurement time required to overcome the large uncertainty in the counting statistics.

Organic scintillators detect both gamma rays and fast neutrons, and can discriminate between the two using pulse shape discrimination (PSD). Neutron detection in organic scintillators mostly relies on elastic scattering on hydrogen nuclei. Because this reaction has a large cross-section for fast neutrons, no moderation is required in systems using these detectors. Consequently, the neutron lifetime within the system is of the order of tens of nanoseconds, which is several orders of magnitude shorter compared to the neutron lifetime in thermal neutron counters. This feature makes it possible to reduce the coincidence window by approximately three orders of magnitude in fast systems, compared to thermal systems, resulting in significantly lower statistical measurement uncertainty $[6,7]$. 
In this work, we report the development and experimental validation of a fast-neutron multiplicity counter (FNMC), based on sixteen organic scintillation detectors. The FNMC was used at Idaho National Laboratory (INL) to passively assay two sets of plutonium metal plates, hereafter referred to as PAHN and PANN. Each plate had a total mass of approximately $100 \mathrm{~g}$, but different isotopic composition (74\% and $95 \%{ }^{239} \mathrm{Pu}$ mass percentage, for PAHN and PANN series, respectively). The overall ${ }^{240} \mathrm{Pu}$-effective $\left({ }^{240} \mathrm{Pu}_{\text {eff }}\right)$ mass of the measured assemblies of plates was in the $4.7 \mathrm{~g}-476 \mathrm{~g}$ range. To quantify the

${ }^{240} \mathrm{Pu}_{\text {eff }}$ mass of the samples, we calibrated the system response using a subset of the results, and then measured the neutron doubles rate emitted by the remaining samples.

\section{Materials and methods}

This section describes the experimental setup used to perform the non-destructive assay of plutonium metal plates at Idhao National Laboratory.

\subsection{Detectors and readout electronics}

The FNMC consists of eight liquid organic scintillators (EJ-309, 7.62-cm diameter by 7.62-cm length, by Eljen, Sweetwater, Tex.) and eight stilbene detectors (5.08-cm diameter by 5.08-cm length, by Lawrence Livermore National Laboratory and Inrad Optics, Northvale, N.J.). The detectors were positioned in an alternating arrangement, so that two detectors of the same type were never next to each other, in the horizontal or in the vertical plane (Fig. 1). EJ-309 and stilbene feature similar elemental composition (Table 1) and are both PSD-capable organic scintillators. PSD performance of the detectors is critical in this application, where fission neutrons from 0.1 to several MeV must be detected in the presence of an
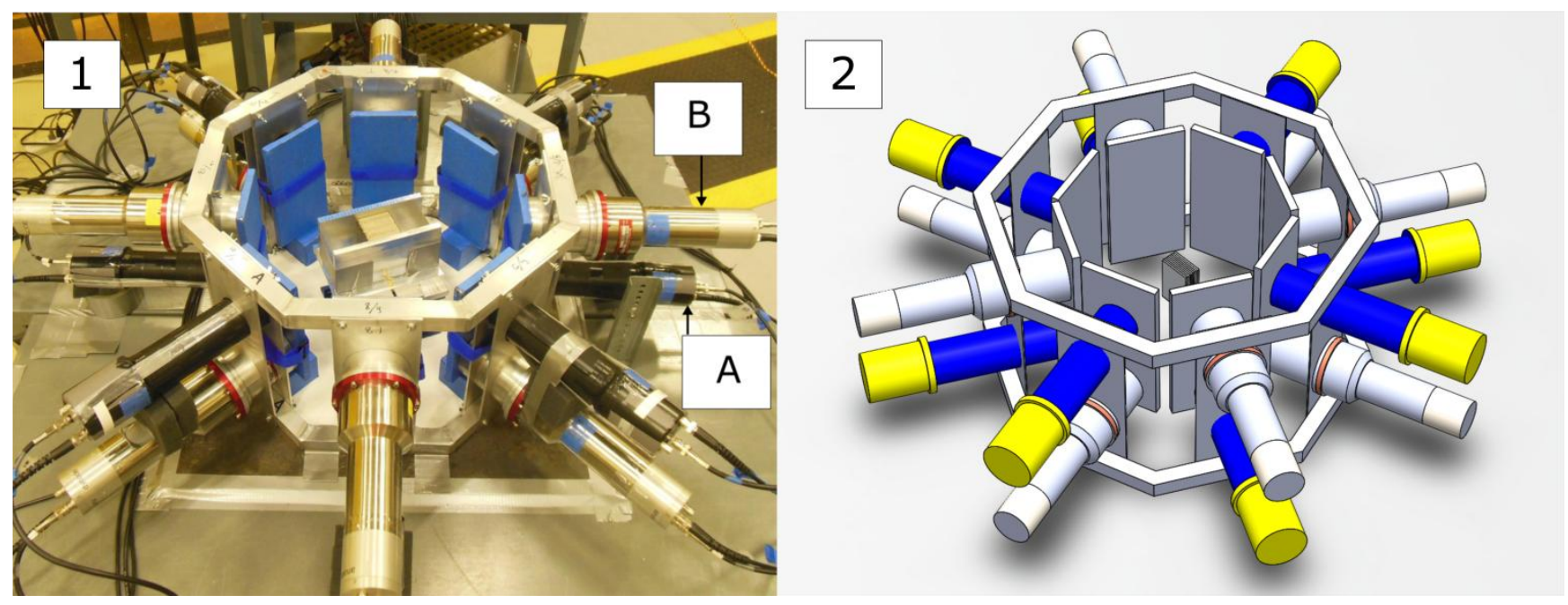

Figure 1. Picture (1) and three-dimensional model (2) of the fast-neutron multiplicity counter based on stilbene (A) and EJ-309 (B) detectors at INL, while measuring 19 plutonium metal plates. 
Table 1. Properties of EJ-309 and stilbene detectors.

59

60

61

62

63

64

\begin{tabular}{ccc}
\hline & EJ-309 [8] & Stilbene \\
\hline Chemical formula & n. a. & $\mathrm{C}_{14} \mathrm{H}_{12}$ \\
\hline Geometric isomerism & n. a. & Trans \\
H:C ratio & 1.25 & 0.86 \\
\hline Light output (\% anthracene) & 80 & $64,69^{*}$ \\
Maximum wavelength (nm) & 424 & 390 \\
Scintillation efficiency & 12300 & $9670^{* *}$ \\
(photons/ 1 MeVee) & 3.5 & $4.5^{* *}$ \\
Fast decay time constant (ns) & 144 & $\mathrm{n}$. a. \\
\hline Flash point ( ${ }^{\circ}$ C) & $7.62 ; 7.62$ & $5.08 ; 5.08$ \\
\hline Size (length, cm; diameter, cm) & $9214 \mathrm{~B} ; \mathrm{ET}$ & $9214 \mathrm{~B} ; \mathrm{ET}$ \\
Photomultiplier tube & Enterprises Ltd & Enterprises Ltd \\
(model; manufacturer) & (Uxbridge, UK) & (Uxbridge, UK) \\
\hline
\end{tabular}

* Relative light output refers to solution-grown stilbene, compared to two anthracene crystals with different structural perfections [9].

70 ** This value refers to melt-grown stilbene [5][10], solution-grown crystal is expected to outperform the scintillation efficiency figure by about 50\%[9].

72 Pulses were acquired and directly converted to digital waveforms using a 500-MS/s, 14-bit, 16-channel 73 digitizer (V1730 by CAEN Technologies, Viareggio, Italy [11]). We performed pulse shape discrimination 74 using a standard charge integration method [12].

75
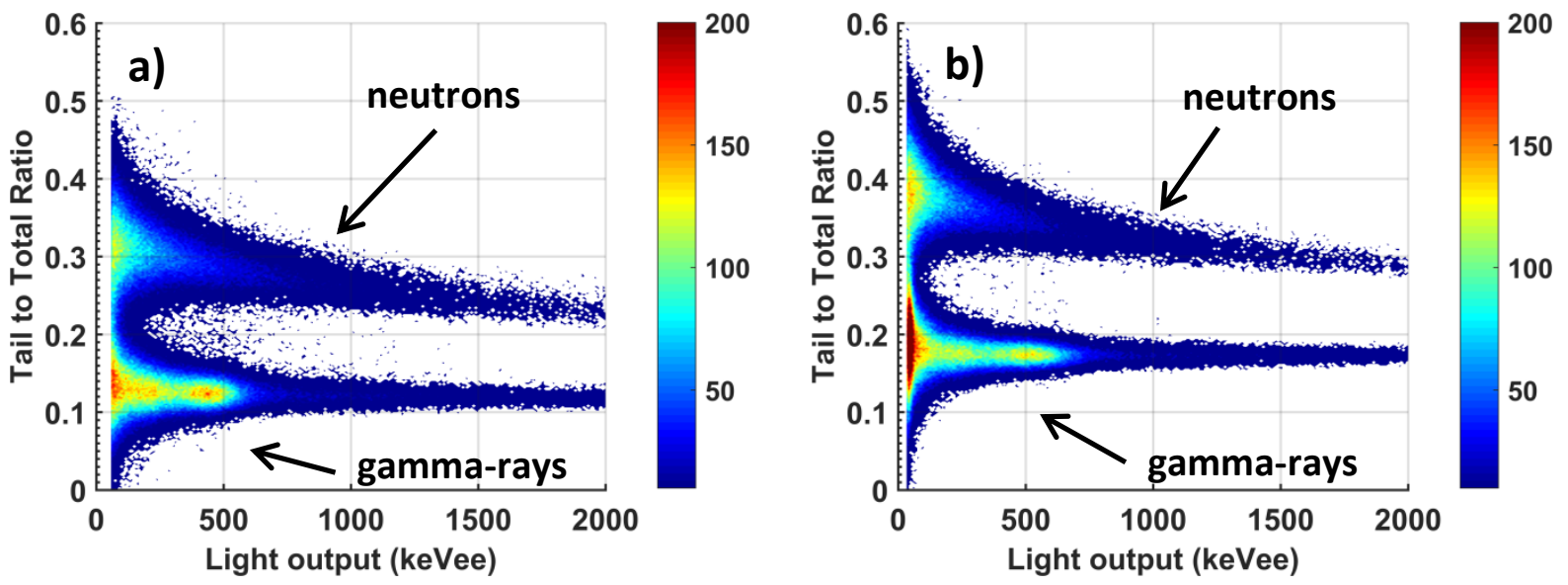

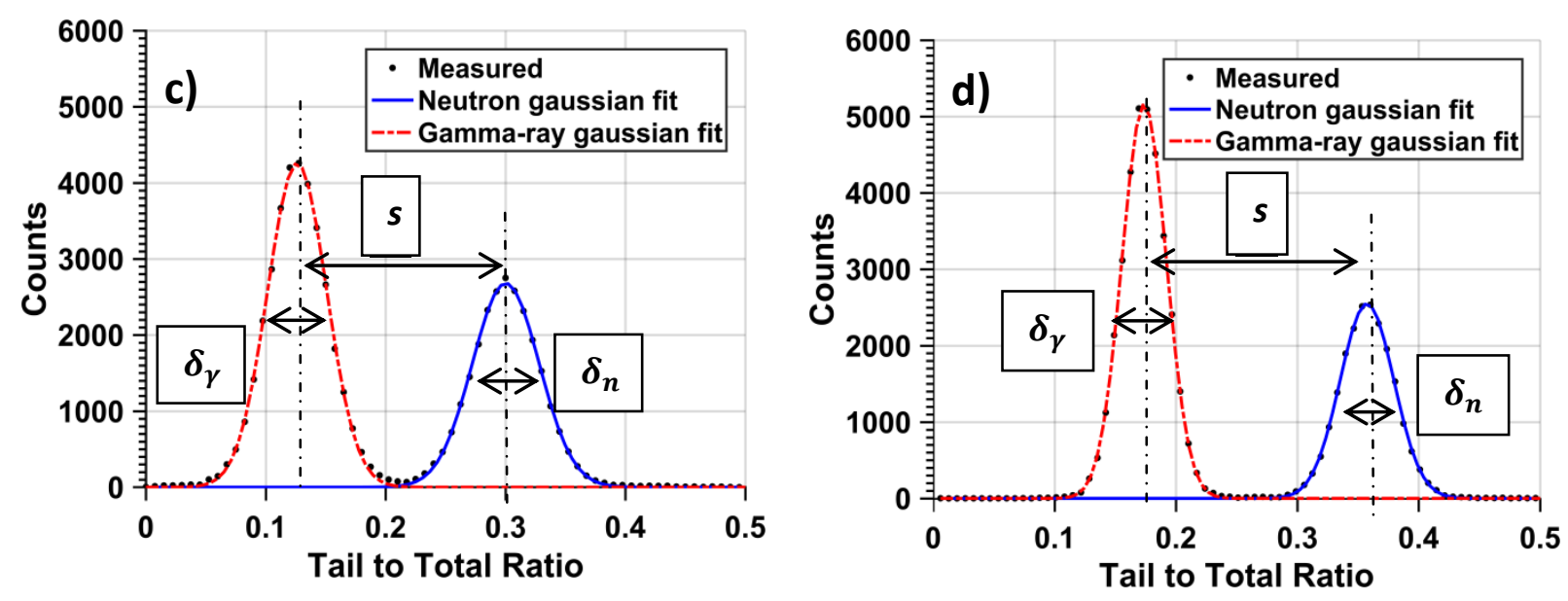

Figure 2. PSD analysis for an EJ-309 (a) and a stilbene detector (b). PSD plots correspond to detector irradiation with one of the plutonium metal samples. Count distribution as a function of the tail to total ratio for 100-200 keVee light output, for EJ-309 (c) and stilbene (d).

This method is based on the delayed-fluorescence component, selectively emitted after scintillator interactions with high-LET charged particles, e.g., recoil protons produced by neutron scattering [13]. Therefore, neutron-induced pulses exhibit an overall slower decay constant, compared to photongenerated pulses. The charge integration method estimates the energy deposited in the tail of each pulse, relative to the total pulse energy, to discriminate between photon and neutron pulses. We found that the tail start time for an optimized PSD was $24 \mathrm{~ns}$ after the pulse peak.

The ratio between the pulse tail and pulse total integral (tail-to-total ratio) as a function of the total pulse integral, in light output units, for $5 \times 10^{5}$ detected pulses, is shown in Fig. 2a and Fig. 2b. A figure of merit (FOM) value is typically used to characterize the scintillator PSD performance. The FOM can be defined as $s /\left(\delta_{n}+\delta_{\gamma}\right)$, where $s$ is the separation between the peaks of the neutron and gamma-ray distributions (Fig 2c-d), at a given light output, and $\delta_{n}$ and $\delta_{\gamma}$ are the full-widths at half-maximum of the neutron and gamma-ray bands, respectively (Fig. 2c, Fig. 2d).

The system achieved a readout rate of approximately $60 \mathrm{MBps}$, with a negligible dead time, corresponding to approximately 140,000 waveforms per second. For some of the plutonium metal assemblies the trigger rate exceeded the maximum readout rate, mainly because of the highlyradioactive ${ }^{241} \mathrm{Am}$ present in the fuel from the decay of ${ }^{241} \mathrm{Pu}$ [14]. Therefore, for those cases we implemented an acquisition-in-coincidence readout logic, which drastically reduced the readout throughput due to random background pulses [15]. This coincidence readout logic accepts only those pulses that occur in coincidence within a window of programmable length (for this application, we used $40 \mathrm{~ns})$. Specifically, although each channel is able to trigger independently, pulses that caused a trigger event will be stored if and only if a majority condition is satisfied, i.e. at least two pulses occur in coincidence in our case. This acquisition-in-coincidence strategy discards uncorrelated detection events. Furthermore, all the pulses detected in coincidence by top-bottom nearest neighbor detectors, e.g. 
detectors " $A$ " and " $B$ " in Fig. 1, were also rejected. This readout method is meant to reject most of the spurious coincidence events referred to as cross-talk events [16], which occur whenever a neutron is scattered from one detector into another and deposits enough energy to be detected in both of them. In order to compare the two acquisition methods, a few assemblies were measured using both the singles and the coincidence acquisition mode. In the case of a single PAHN case, for example, the count rate in coincidence mode was approximatively $1 \%$ and $0.08 \%$ of the count rate in singles mode, for neutrons and photons, respectively.

Prior to the experiment, the detectors were gain-matched using a $0.1-\mu C i^{137} \mathrm{Cs}$ source. We used a 25 $\mu C i{ }^{252} \mathrm{Cf}$ source to optimize the detection threshold. It is desirable to operate the detectors at a low energy threshold to maximize the neutron-detection efficiency. However, as the threshold decreases, the number of gamma-ray pulses that are misclassified as neutron pulses unavoidably increases. Considering this effect, we selected a detection threshold such that the misclassification rate of gammaray pulses was about $10^{-6}$ per detection [12]. Due to the superior PSD performance of stilbene, the detection threshold of the stilbene detectors was set to $30 \mathrm{keVee}$, lower than the 50 -keVee threshold used for the EJ-309 detectors. These threshold settings provided a dynamic range of 30-2,400 keVee (this corresponds to approximately 336-6,500 keV neutron energy deposited) for stilbene and 50-2,400 keVee (approximately 520-5,530 keV neutron energy deposited) for EJ-309. The maximum energy deposited by a neutron scattering on hydrogen nuclei was derived using the Voltz model of the light output function, fitted to EJ-309 and stilbene data by Norsworthy and colleagues [17]. The selected readout range minimized the amount clipped pulses, while also amplifying low-amplitude pulses, to improve PSD performance at low energies (<200 keVee).

\subsection{Assay samples: plutonium metal plates}

Table 2 gives the isotopic composition of the two sets of metal plates [18], aged to the date of the experiment, August 2015. These samples are fuel plates designed and built for the INL Zero Power Physics Reactor (ZPPR). The external dimensions of a single plate are $7.62 \mathrm{~cm}$ by $5.08 \mathrm{~cm}$ (thickness $0.3175 \mathrm{~cm}$ ). The plutonium metal core is encapsulated in a thin $304 \mathrm{~L}$ stainless steel cladding. Based on the fuel element geometry [19], we estimate core densities of $15.08 \mathrm{~g} / \mathrm{cm}^{3}$ and $15.09 \mathrm{~g} / \mathrm{cm}^{3}$, for PAHN and PANN.

The ${ }^{240} \mathrm{Pu}_{\text {eff }}$ mass is defined as the mass of ${ }^{240} \mathrm{Pu}$ that would give the same response in terms of neutron coincidences (doubles) as that obtained by the actual ${ }^{238} \mathrm{Pu},{ }^{240} \mathrm{Pu}$ and ${ }^{242} \mathrm{Pu}$ content of the sample. The ${ }^{240} \mathrm{Pu}_{\text {eff }}$ mass for systems based on ${ }^{3} \mathrm{He}$ detectors is calculated as a linear combination of the masses of the plutonium isotopes having even mass numbers, $A$ (Eq. 1 ). The neutron coincidence rate is used to estimate the sample ${ }^{240} \mathrm{Pu}_{\text {eff }}$ mass and depends on both the multiplicity distribution of the sample and the efficiency of the system to detect multiplets of the second-order (i.e. neutron doubles). For this reason, the multiplicative coefficients in Eq. 1 are expected to be different for fast and thermal systems. Dolan and colleagues simulated the neutron coincidence response of a fast-neutron multiplicity counter based on 16 EJ-309 scintillators [20], very similar to our FNMC, and derived the ${ }^{240} \mathrm{Pu}_{\text {eff }}$ mass equation coefficients, specific to their system. The PAHN and PANN ${ }^{240} \mathrm{Pu}_{\text {eff }}$ mass obtained using these coefficients was not significantly different from the one calculated using the coefficients for thermal systems (Eq. 1), 
which were then used in this work. The ${ }^{240} \mathrm{Pu}_{\text {eff }}$ mass for a single PANN and PAHN plate is $4.70 \mathrm{~g}$ and 25.05 g, respectively.

$$
{ }^{240} P u_{e f f}=2.49{ }^{238} P u+{ }^{240} P u+1.57{ }^{242} P u
$$

Table 2. Isotopic composition of PAHN and PANN plates, August 2015. Isotope mass uncertainty was calculated taking into account the known uncertainty in the half-life of each isotope and the aging time.

\begin{tabular}{ccc}
\hline & PAHN mass (g) & PANN mass (g) \\
\hline${ }^{238} \mathrm{Pu}$ & $0.00020(7)$ & $0.00023(6)$ \\
${ }^{239} \mathrm{Pu}$ & $79.69(2)$ & $98.89(2)$ \\
${ }^{240} \mathrm{Pu}$ & $23.92(1)$ & $4.70(1)$ \\
${ }^{241} \mathrm{Pu}$ & $0.65(1)$ & $0.04(1)$ \\
${ }^{242} \mathrm{Pu}$ & $0.67(1)$ & $<0.009$ \\
${ }^{241} \mathrm{Am}$ & $1.87(1)$ & $0.23(1)$ \\
$\mathrm{Al}$ & 1.25 & 1.16 \\
Total plutonium & 106.79 & 103.87 \\
\hline
\end{tabular}

Actinide decay in the PAHN and PANN fuels produces overall gamma-ray emission rates of $8.1 \times 10^{10} \mathrm{~s}^{-1}$ and $1.1 \times 10^{10} \mathrm{~s}^{-1}$, respectively. The higher gamma-ray activity of PAHN plates, with respect to PANN, is due to its higher ${ }^{241} \mathrm{Pu}$ and ${ }^{240} \mathrm{Pu}$ mass content, and thus higher ${ }^{241} \mathrm{Am}$ content [14]. Although the energy of these gamma rays is relatively low $(<700 \mathrm{keV})$, their intensity may be problematic, especially when lowering the energy threshold of the scintillators to increase the neutron detection efficiency. The neutron emission rates for the PAHN and PANN are $1.17 \times 10^{4}$ and $2.20 \times 10^{3} \mathrm{~s}^{-1}$, respectively. Singles acquisition mode was used for PANN, while coincidence mode was used for PAHN. The gammaray/neutron ratio detected by the scintillators, which are shielded by $1.2 \mathrm{~cm}$-thick lead plates (Fig. 1), is about 65 for the PAHN and 40 for the PANN in singles mode, and approximately 4 in coincidence mode, for both types of plate. These gamma-ray/neutron ratios were calculated after rejecting clipped and piled-up pulses, which accounted for about $1 \%$ of the total acquired pulses. 

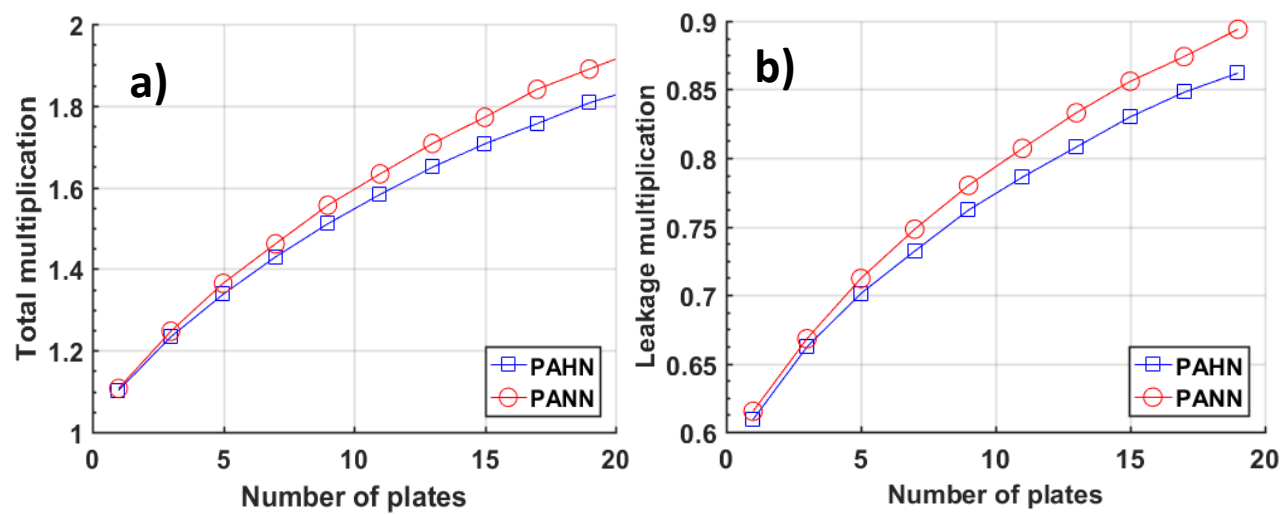

Figure 3. Simulated total multiplication (a) and leakage multiplication (b) for PAHN and PANN plate assemblies of 1-19 plates.

A multiplicity counter is sensitive to the multiplicity of the fission events. The neutron multiplication $M$ characterizes the dynamics of the fission neutron population of a finite multiplying system. The neutron multiplication is calculated as the number of neutrons in the sample, divided by the first-generation neutron population. The neutron multiplication was simulated for subcritical assemblies of up to 19 plates of PAHN and PANN plates (Fig. 3a). The leakage multiplication, $M_{L}$, is the measureable multiplication and it is defined as the ratio between the number of neutrons leaving the sample and the number of neutrons produced within the sample, by spontaneous and induced fission in the case of plutonium metal samples (Fig. 3b).

The ${ }^{240} \mathrm{Pu}_{\text {eff }}$ mass of a sample depends not only on its mass, but also on the multiplication. Samples with the same mass and elemental composition, but with different multiplication due to, for example, a different geometric configuration, may yield a different ${ }^{240} \mathrm{Pu}_{\text {eff }}$ mass. The current system operates in coincidence mode: ${ }^{240} \mathrm{Pu}_{\text {eff }}$ mass estimate relies on the rate of neutron pairs detected in coincidence. Therefore, the estimate of ${ }^{240} \mathrm{Pu}_{\text {eff }}$ mass for samples with different mass and multiplication poses an undetermined problem. It is therefore necessary to either correct for the neutron multiplication [1], or to derive, experimentally or via simulation, a system response curve, in terms of ${ }^{240} \mathrm{Pu}_{\text {eff }}$ mass, for each sample composition to be assayed. In this work, we demonstrate both approaches.

\subsection{Single-parameter calibration procedure and uncertainty estimation}

The coincidence counting system was first calibrated in single-parameter mode by measuring the coincidence rate, $D$, as a function of ${ }^{240} \mathrm{Pu}_{\text {eff }}$ mass, for samples of known mass. The following procedure was used to derive the calibration curve and the uncertainty value associated to the estimated ${ }^{240} \mathrm{Pu}_{\text {eff }}$ mass of unknown samples.

The most appropriate fit for the data was a polynomial relationship (Eq. 2). The least-squares fitting process gives an estimate of the model coefficients, $a$ and $b$, and their variances: $s_{\mathrm{a}}{ }^{2}$ and $s_{\mathrm{b}}{ }^{2}[21]$.

$D=a{ }^{240} P u_{e f f}^{2}+b{ }^{240} P u_{e f f}$

The ${ }^{240} \mathrm{Pu}_{\text {eff }}$ mass can be related to the measured coincidence count rate using the following expression (Eq. 3), once the calibration curve is established. 
$184 \quad{ }^{240} P u_{e f f}=\frac{\left(\sqrt{4 a D+b^{2}}-b\right)}{2 a}$

185 An uncertainty, $u$, can be assigned to the estimated mass by propagating the uncertainty through Eq. 3. 186 The primary assumption is that each neutron double (pair of neutrons detected in coincidence) is the 187 result of a single fission reaction and thus the uncertainty associated with the number of measured 188 doubles can be modeled as a Poisson process and the variance of the count rate $\left(s_{D}{ }^{2}\right)$ is equal to its 189 mean.

$u^{2}=\left(\frac{\partial^{240} P u_{e f f}}{\partial D}\right)^{2} s_{D}^{2}+\left(\frac{\partial^{240} P u_{e f f}}{\partial a}\right)^{2} s_{a}^{2}+\left(\frac{\partial^{240} P u_{e f f}}{\partial b}\right)^{2} s_{b}^{2}+2\left(\frac{\partial^{240} P u_{e f f}}{\partial a}\right)\left(\frac{\partial^{240} P u_{e f f}}{\partial b}\right) s_{a b}$

191 In Eq. 4, $s_{a b}$ is the covariance between the two coefficients $a$ and $b$.

\subsection{Two-parameter calibration procedure and uncertainty estimation}

193 The two-parameter calibration approach aims at removing the effect of multiplication from the assay. 194 We constructed a calibration curve by plotting the ratio of double-to-single neutron count rate (D/S), as 195 a function of neutron coincidence rate per gram of ${ }^{240} \mathrm{Pu}_{\text {eff }}$ mass $\left(D /{ }^{240} \mathrm{Pu}_{\text {eff }}\right)$. Because both $\mathrm{D} / \mathrm{S}$ and $196 \mathrm{D} /{ }^{240} \mathrm{Pu}_{\text {eff }}$ depend on the sample multiplication, their relationship is expected to be linear (Eq. 5) for a 197 series of samples with the same contribution of $(\alpha, n)$ neutrons, which is estimated to be zero for the 198 INL plutonium metal plates.

$199 \quad \frac{D}{S}=c \frac{D}{240 P u_{e f f}}+d$

200 After deriving the calibration curve, we measured $D / S$ for the samples to be assayed and determined 201 their neutron coincidence rate per unit ${ }^{240} \mathrm{Pu}_{\text {eff }}$ mass $\left(K=\frac{D}{{ }^{240} \mathrm{Pu}_{e f f}}\right)$ from the calibration curve. Because $202 D$ can be measured, the value of ${ }^{240} \mathrm{Pu}_{\text {eff }}$ mass was simply calculated as $D / K$.

203 The uncertainty to be associated with the estimated ${ }^{240} \mathrm{Pu}_{\text {eff }}$ mass, $u$, is then derived using Eq. 6, with 204 analogous assumptions and procedures as for Eq. 4.

$205 u^{2}=$

$206 \quad\left(\frac{\partial^{240} P u_{e f f}}{\partial D}\right)^{2} s_{D}^{2}+\left(\frac{\partial^{240} P u_{e f f}}{\partial S}\right)^{2} s_{S}^{2}+\left(\frac{\partial^{240} P u_{e f f}}{\partial c}\right)^{2} s_{c}^{2}+\left(\frac{\partial^{240} P u_{e f f}}{\partial d}\right)^{2} s_{d}^{2}+$

$2072\left(\frac{\partial^{240} P u_{e f f}}{\partial c}\right)\left(\frac{\partial^{240} P u_{e f f}}{\partial d}\right) s_{c d}$

208 In Eq. $6, s^{2}{ }_{c}$ and $s^{2}{ }_{d}$ are the variances associated with the calibration curve parameters, $c$ and $d$, 209 respectively, and $s_{c d}$ is their covariance coefficient. 


\section{Results}

\section{$212 \quad 3.1$ Comparison of EJ-309 and stilbene PSD performance}

213 Fig. 4 shows the FOM as a function of light output for pulses acquired for an assembly of 19 PANN plates, for a stilbene and an EJ-309 detector. The FOM in Fig. 4 is calculated from scatter plots reported in Fig. 2.a-b. The separation between the gamma-ray and neutron regions increases with light output, generating an increasing trend in the FOM. The FOM is higher in the stilbene detector than in the EJ-309 liquid scintillation detector. This excellent stilbene PSD performance allows us to operate the detector at

218 a threshold as low as 30 keVee.
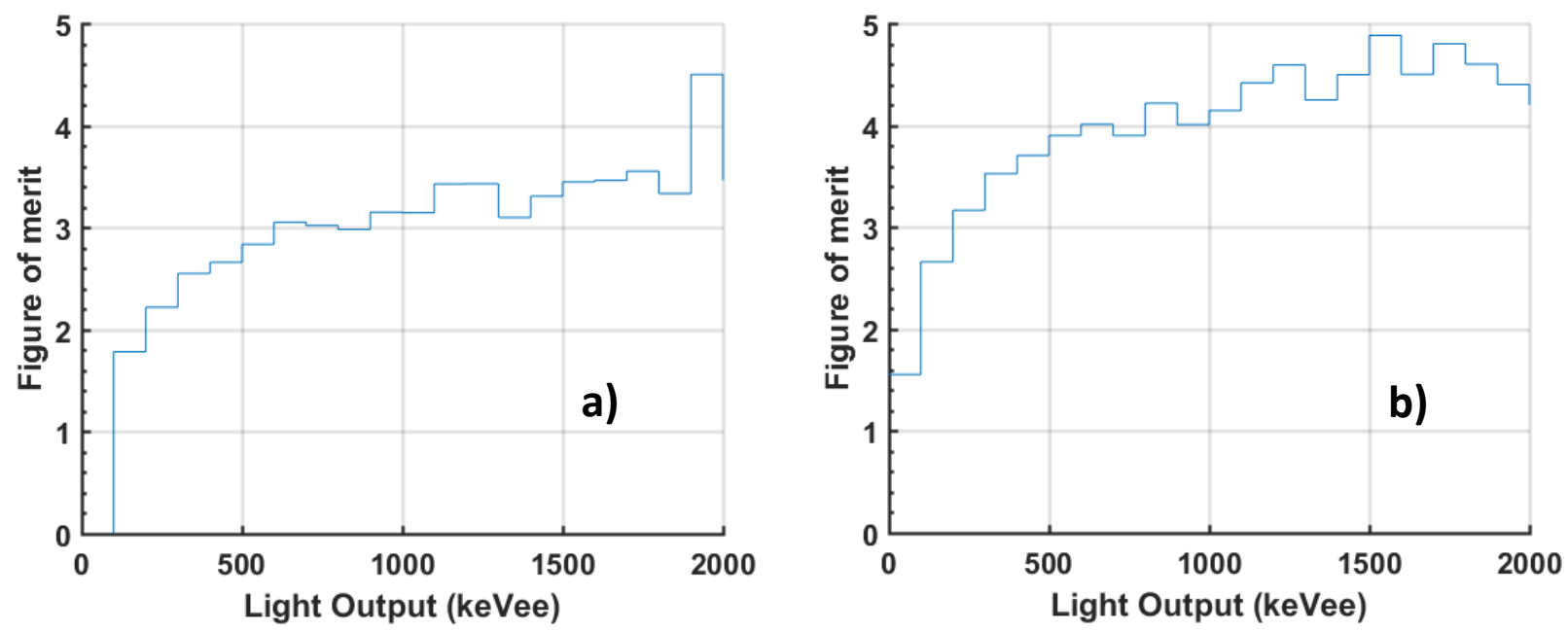

Figure 4. Figure-of-merit for an EJ-309 (a) and a stilbene detector (b), irradiated using 19 PANN plates.

Fig. 5 shows the count rate of neutron doubles as a function of ${ }^{240} \mathrm{Pu}_{\text {eff }}$ mass. Neutrons detected in coincidence by 8 EJ-309 and 8 stilbene detectors are shown separately. The lower absolute response of stilbene crystals, compared to EJ-309 detectors (Fig. 5a-c), is due to their smaller volume. However, the stilbene response, normalized by detector volume and detection solid angle, is consistently higher than the EJ-309 response by about 50\% (Fig. 5b-d). The higher normalized detection efficiency of stilbene, compared to EJ-309, is due to the detection threshold, which is lower in stilbene (30 keVee), than in EJ309 (50 keVee). If the scintillators were to be operated at the same threshold (Fig. 5b-d) the stilbene overall response would be lower than EJ-309 because of its lower light output (Table 2), negatively affected by quenching phenomena of the proton pulses [13]. 

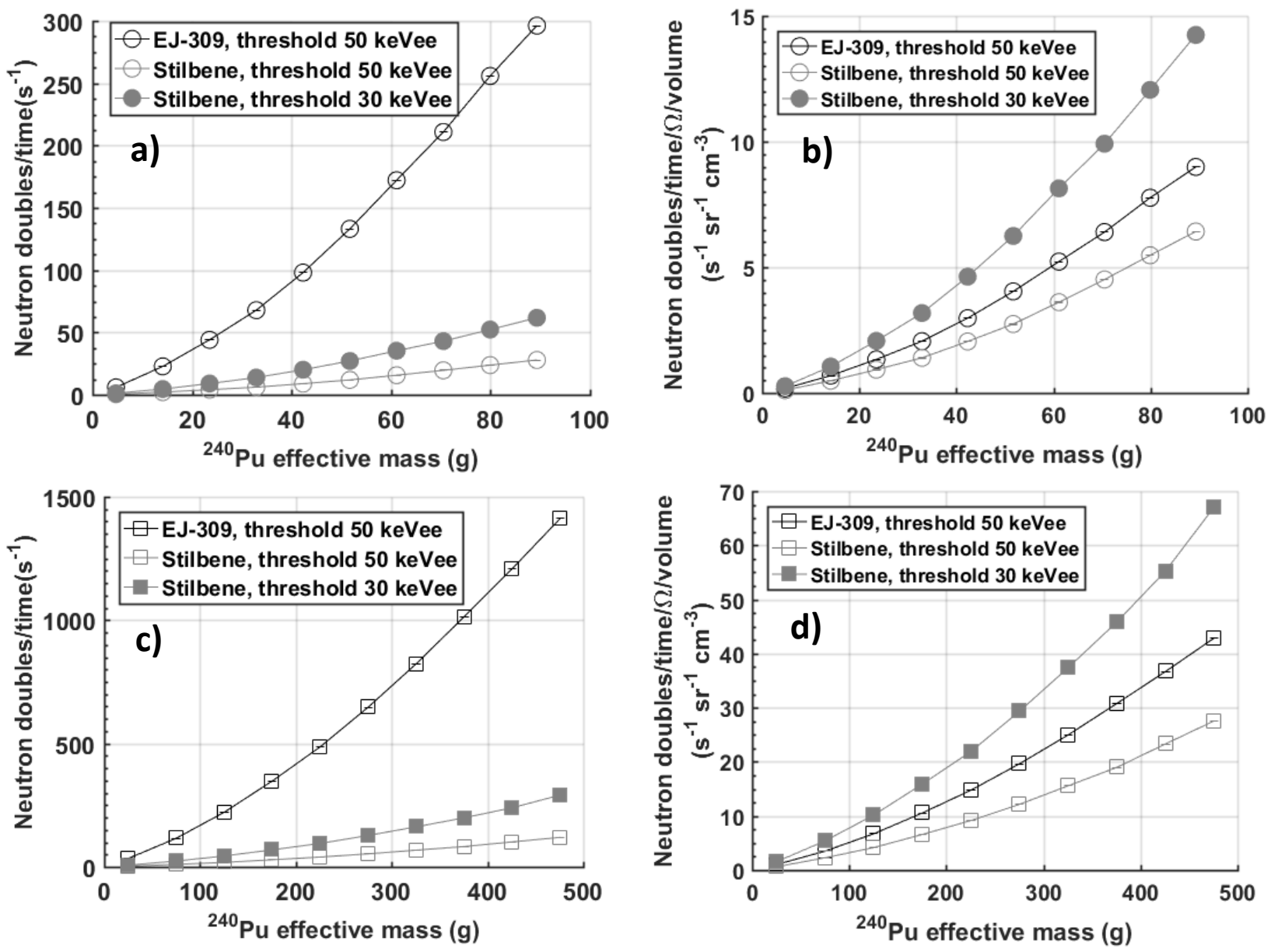

Figure 5. Count rate of neutron doubles per unit solid angle and volume as a function of the number of plates (PANN series: $a, b$ and PAHN series: $c, d)$. Stilbene and EJ309 contributions are separated $(a, c)$ and normalized by volume and solid angle $(b, d)$.

\subsection{System simulation and cross-talk rejection}

A model of the plutonium metal samples and the FNMC was developed in MCNPX-PoliMi [22] in preparation of the experiment. Fig. 6 shows the measured and simulated neutron multiplet rates for the two sets of plates. The goal of the simulation was to find the optimal detector-to-sample distance, sample orientation, and lead thickness to maximize the sensitivity to emitted neutron doubles as well as characterize the gamma-ray response. We used the MCNPX-PoliMi built-in spontaneous fission ${ }^{240} \mathrm{Pu}$ model, which simulates the energy distribution of the emitted neutrons as a function of the multiplicity, as well as an anisotropic angular distribution. The simulated and measured double rates generally agree well, while higher order simulated multiplets tend to overestimate the measurement. The effect of rejecting coincidence counts from top-bottom nearest neighbor detectors resulted in a decrease of the overall response by $8.9 \%$ and was considered in the simulation.

In principle, a neutron coincidence event may occur after a spallation reaction between high-energy charged particles, produced by cosmic rays, and the nuclei of a high-Z material. This effect was 
242 estimated to be negligible in our system. The rate of background double neutrons was measured as $<10^{-}$

$243{ }^{2} \mathrm{~s}^{-1}$ in the ZPPR facility building; therefore, no background correction was applied.
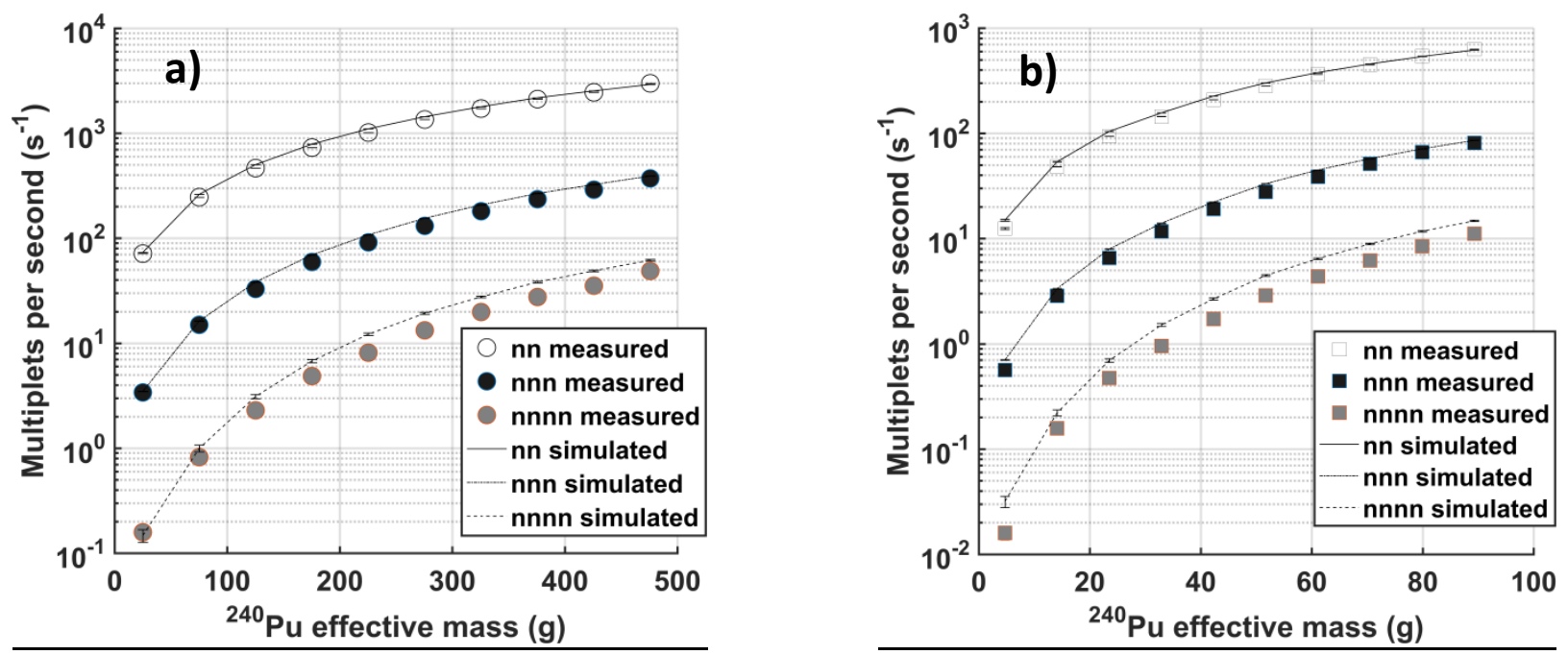

Figure 6. Simulated and measured rate of neutron multiplets as a function of ${ }^{240} \mathrm{Pu}_{\text {eff }}$ mass, for PAHN (a) and PANN plates (b).

\subsection{Estimate of ${ }^{240} \mathrm{Pu}$ effective mass using single-parameter calibration}

Assemblies of 3, 9 and 15 plates were used to build a calibration curve for each data set (Fig. 7a and Fig.8a). Assemblies of $1,5,7,11,13,17$, and 19 plates were used as a test set; their mass was assumed to be unknown and was estimated using Eq. 3. The process of measuring the unknown ${ }^{240} \mathrm{Pu}_{\text {eff }}$ mass of sample is exemplified by two arrows in Fig. 7b, for an assembly of 13 PANN plates. Fig. 7b and Fig. 8b show the estimated ${ }^{240} \mathrm{Pu}_{\text {eff }}$ mass using the single-parameter calibration procedure, compared to the known ${ }^{240} \mathrm{Pu}_{\mathrm{eff}}$ mass for PANN and PAHN plate assemblies, respectively.

254 Table 3 shows the estimated values of ${ }^{240} \mathrm{Pu}_{\text {eff }}$ mass for the test-set plate assemblies and the uncertainties associated with them, $u$. The uncertainty was calculated using the technique in the previous section, and accounts for counting uncertainty and possible deficiencies of the fitting model in

257 capturing all the information in the data. 

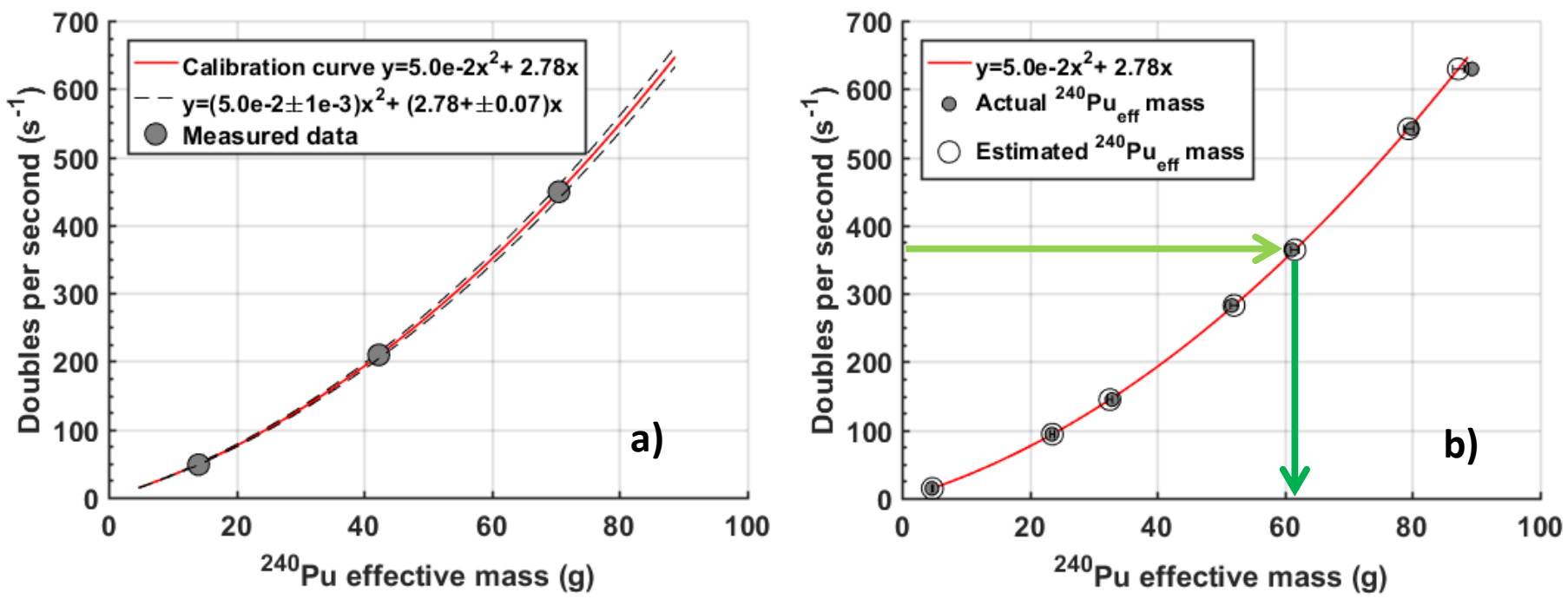

Figure 7. Fit of neutron doubles rate as a function of ${ }^{240} \mathrm{Pu}_{\text {eff }}$ mass to build the calibration curve (a) and validation of the calibration curve using assemblies of $1,5,7,11,13,17$, and 19 plates (b). Both graphs refer to PANN plates.

259 We also report the relative bias of the estimated ${ }^{240} \mathrm{Pu}_{\text {eff }}$ mass with respect to the known ${ }^{240} \mathrm{Pu}_{\text {eff }}$ mass.

260 This relative difference should account for all the sources of systematic errors, including any drift in the 261 system working conditions, which could systematically affect the measurement results. The bias error is 262 always within $\pm 3 u$, which proves that no significant bias can be identified during the system operation 263 and the model explains well the intrinsic measurement uncertainty of the system.
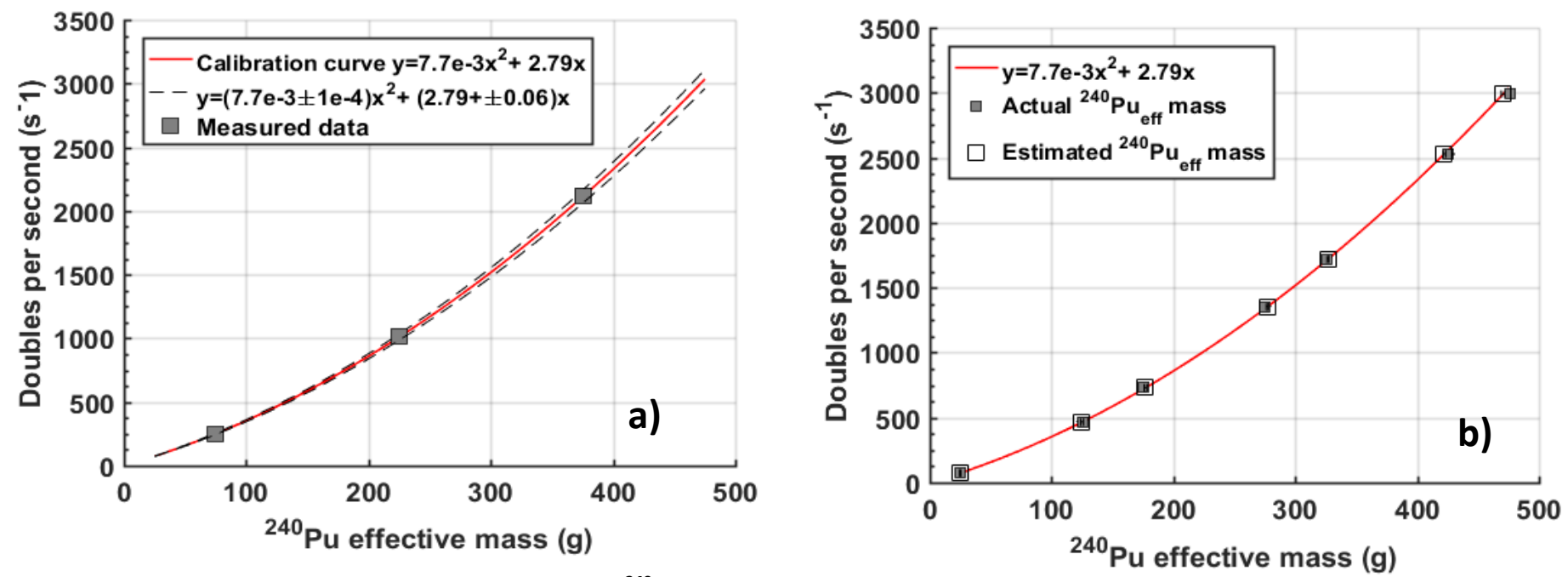

Figure 8. Fit of neutron doubles rate as a function of ${ }^{240} \mathrm{Pu}_{\text {eff }}$ mass to build the calibration curve (a) and validation of the calibration curve using assemblies of 1, 5, 7, 11, 13, 17, and 19 plates (b). Both graphs refer to PAHN plates.

265 We post-processed a subset of the data to study the dependence of the measurement statistical 266 uncertainty as a function of the assay time. An uncertainty lower than $1 \%$ in the ${ }^{240} \mathrm{Pu}_{\text {eff }}$ mass was 
267

268

269

270

271

272

273

274

275

276

277

278

achieved in a 4-minute assay time, for ${ }^{240} \mathrm{Pu}_{\text {eff }}$ masses higher than $50 \mathrm{~g}$, as shown in Fig. 9. The percentage uncertainty is expected to be higher for smaller samples.

In a 4-minute assay, our system estimated a ${ }^{40} \mathrm{Pu}_{\text {eff }}$ mass of $4.78 \pm 0.11 \mathrm{~g}$ and $23.58 \pm 0.25 \mathrm{~g}(2.4 \%$ and $1.1 \%$ relative uncertainty, respectively) for PANN assemblies of one and three plates, respectively, whose declared ${ }^{40} \mathrm{Pu}_{\text {eff }}$ masses were $4.70 \pm 0.02 \mathrm{~g}$ and $23.50 \pm 0.02 \mathrm{~g}$, respectively. For comparison to the currently used thermal systems, the High Level Neutron Coincidence Counter II (HLNCC-II) is able to estimate a ${ }^{40} \mathrm{Pu}_{\text {eff }}$ mass of $9.70 \pm 0.39 \mathrm{~g}$ (4.0\% relative uncertainty) for a plutonium metal sample of 9.39-g declared ${ }^{40} \mathrm{Pu}_{\text {eff }}$ mass, in 5.5 minutes [6]. A lower percent uncertainty of $1.3 \%$ is achieved for the same sample in the HLNCC-II by increasing the assay time to 110 minutes. The HLNCC-II consists of a single ring of 18 cylindrical ${ }^{3} \mathrm{He}$ proportional detectors $(2.5 \mathrm{~cm}$ diameter by $50 \mathrm{~cm}$ length) filled to a pressure of $4 \mathrm{~atm}$.

Table 3. Estimated ${ }^{240} \mathrm{Pu}_{\text {eff }}$ mass using the single-parameter calibration method.

\begin{tabular}{|c|c|c|c|c|c|c|c|}
\hline \multicolumn{8}{|c|}{ PAHN } \\
\hline $\begin{array}{l}\text { Number of } \\
\text { plates }\end{array}$ & 1 & 5 & 7 & 11 & 13 & 17 & 19 \\
\hline $\begin{array}{l}\text { Actual }{ }^{240} \mathrm{Pu} \\
\text { effective mass } \\
\text { (g) }\end{array}$ & 25.05 & 125.23 & 175.32 & 275.51 & 325.60 & 425.79 & 475.88 \\
\hline $\begin{array}{l}\text { Estimated } \\
{ }^{240} \mathrm{Pu} \text { effective } \\
\text { mass (g) }\end{array}$ & $\begin{array}{r}24.92 \\
\pm 0.49\end{array}$ & $\begin{array}{c}124.98 \\
\pm 1.13\end{array}$ & $\begin{array}{c}177.10 \\
\pm 1.08\end{array}$ & $\begin{array}{c}276.99 \\
\pm 0.74\end{array}$ & $\begin{array}{c}326.75 \\
\pm 0.66\end{array}$ & $\begin{array}{c}422.30 \\
\pm 1.09\end{array}$ & $\begin{array}{c}470.53 \\
\pm 1.49\end{array}$ \\
\hline $\begin{array}{l}\text { Absolute bias } \\
\text { (g) }\end{array}$ & -0.12 & -0.25 & 1.78 & 1.48 & 1.15 & -3.49 & -5.35 \\
\hline \multicolumn{8}{|c|}{ PANN } \\
\hline $\begin{array}{l}\text { Number of } \\
\text { plates }\end{array}$ & 1 & 5 & 7 & 11 & 13 & 17 & 19 \\
\hline $\begin{array}{l}\text { Actual }{ }^{240} \mathrm{Pu} \\
\text { effective mass } \\
\text { (g) }\end{array}$ & 4.70 & 23.50 & 32.90 & 51.71 & 61.11 & 79.91 & 89.31 \\
\hline $\begin{array}{l}\text { Estimated } \\
{ }^{240} \mathrm{Pu} \text { effective } \\
\text { mass (g) }\end{array}$ & $\begin{array}{c}4.78 \\
\pm 0.10\end{array}$ & $\begin{array}{l}23.43 \\
\pm 0.24\end{array}$ & $\begin{array}{l}32.45 \\
\pm 0.22\end{array}$ & $\begin{array}{l}51.48 \\
\pm 0.16\end{array}$ & $\begin{array}{l}61.46 \\
\pm 0.15\end{array}$ & $\begin{array}{l}80.25 \\
\pm 0.21\end{array}$ & $\begin{array}{l}88.79 \\
\pm 0.26\end{array}$ \\
\hline $\begin{array}{l}\text { Absolute bias } \\
\text { (g) }\end{array}$ & 0.08 & -0.07 & -0.46 & -0.22 & 0.35 & 0.34 & -0.52 \\
\hline
\end{tabular}



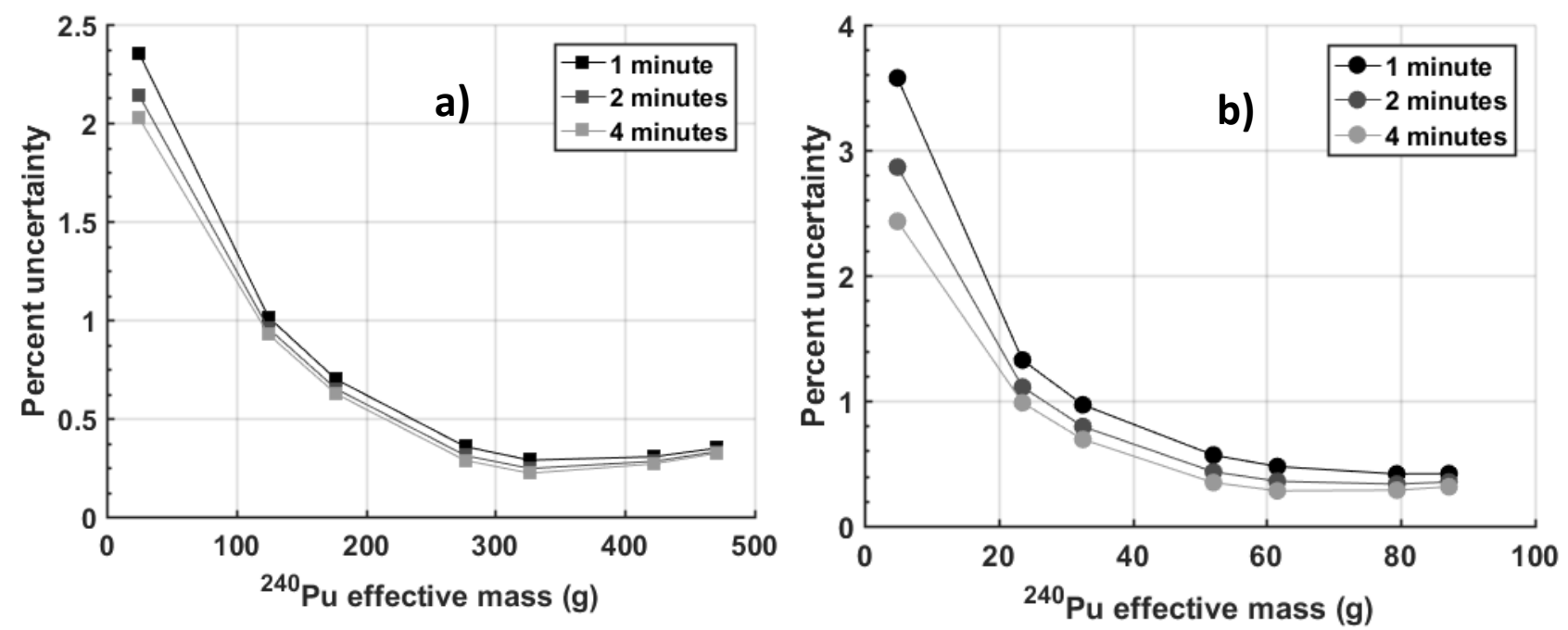

Figure 9. Statistical percent uncertainty as a function of ${ }^{240} \mathrm{Pu}_{\text {eff }}$ mass for increasing assay times, for PAHN (a) and PANN plates (b).

\subsection{Comparison of EJ-309 and stilbene detectors in terms of measurement precision}

We have evaluated the contribution to the overall measurement uncertainty given by 8 EJ-309 and 8 stilbene detectors, considered as two separate arrays. Measurement uncertainty is affected by two main components, the counting statistics (first term in Eq. 4) and the goodness of the fitted model (second, third and fourth term in Eq. 4). We analyzed these contributions separately, for stilbene and EJ309 detectors for a 4-minute assay of the PANN plate series (Fig. 10).

In this case, the uncertainty is dominated by the counting statistics component (Fig. 10). For this reason, the array of EJ-309 detectors yields an overall lower uncertainty, because of the larger volume and thus higher intrinsic efficiency of EJ-309 detectors, compared to stilbene crystals.
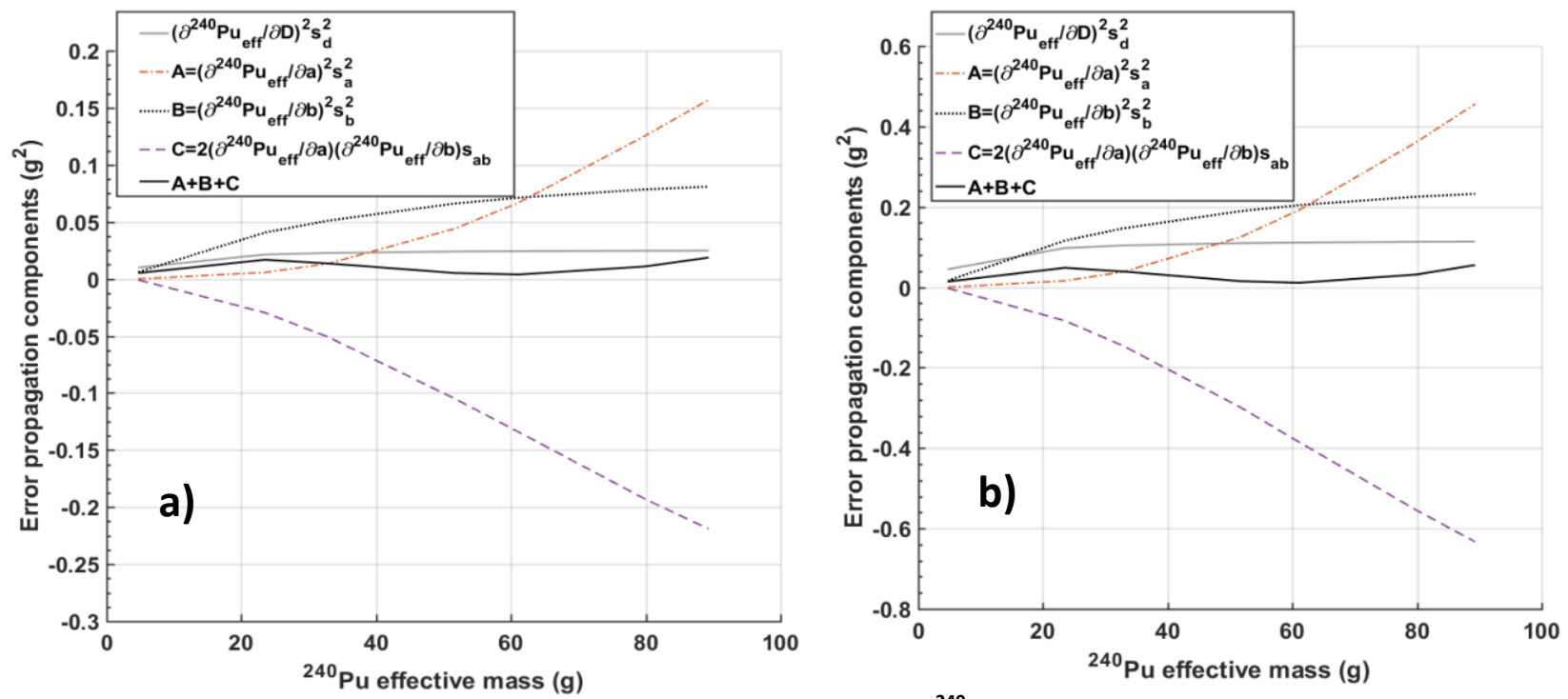

Figure 10. Components of the error propagation (Eq. 4), as a function of ${ }^{240} \mathrm{Pu}_{\text {eff }}$ mass, for pairs of EJ-309 (a) and stilbene detectors (b), for PANN plates and a measurement time of 10 minutes. The solid and black grey lines show the counting statistics and goodness of the fit contributions to the overall uncertainty, respectively. 
Table 4. Comparison of estimated ${ }^{240} \mathrm{Pu}_{\text {eff }}$ mass and its uncertainty by using EJ-309 and stilbene detectors separately, for PAHN plates.

\begin{tabular}{lccccccc}
\hline Number of plates & $\mathbf{1}$ & $\mathbf{5}$ & $\mathbf{9}$ & $\mathbf{1 1}$ & $\mathbf{1 5}$ & $\mathbf{1 7}$ & $\mathbf{1 9}$ \\
\hline $\begin{array}{l}\text { Actual } \\
\text { mass }(\mathbf{g})\end{array}$ & 25.05 & 125.23 & 225.42 & 275.51 & 375.69 & 425.79 & 475.88 \\
$\begin{array}{l}\text { Estimated }{ }^{240} \mathrm{Pu} \\
\text { effective mass - }\end{array}$ & 24.97 & 125.98 & 178.03 & 277.59 & 327.81 & 428.00 & 478.10 \\
$\begin{array}{l}\text { stilbene array (g) } \\
\text { Estimated }{ }^{240} \mathrm{Pu}\end{array}$ & \pm 0.57 & \pm 0.99 & \pm 1.05 & \pm 1.11 & \pm 1.13 & \pm 1.15 & \pm 1.18 \\
$\begin{array}{l}\text { effective mass - EJ-309 } \\
\text { array (g) }\end{array}$ & \pm 0.74 & \pm 1.68 & \pm 1.60 & \pm 1.14 & \pm 1.04 & \pm 1.60 & \pm 2.12 \\
\hline
\end{tabular}

The uncertainty contribution due to counting statistics becomes negligible when assaying samples with activity higher than PANN, or by increasing the measurement time. As we verified for PAHN plates (Table 4), when the number of measured doubles exceeds approximately 25,000, the main source of uncertainty becomes the third term in Eq. 4, which depends on the rate of neutron doubles, the coefficients $a$ and $b$ and the variance, $s^{2}$. We found that in these conditions the ${ }^{240} \mathrm{Pu}_{\text {eff }}$ mass was estimated more precisely by the array of stilbene crystals than EJ-309 detectors, mainly due to a more precise estimate of the $b$ coefficient, whose uncertainty was $0.5 \%$ and $3.1 \%$ for stilbene and EJ-309 detectors, respectively.

\subsection{Estimate of ${ }^{240} \mathrm{Pu}$ effective mass using two-parameter calibration}

We used PANN assemblies of 3,13 and 17 plates as calibration set to test the two-parameter calibration method. As expected, the ratio between double and single neutrons for both sets of plates is well described by a linear relationship as a function of the neutron doubles rate per unit ${ }^{240} \mathrm{Pu}_{\text {eff }}$ mass (Fig. 308 11).
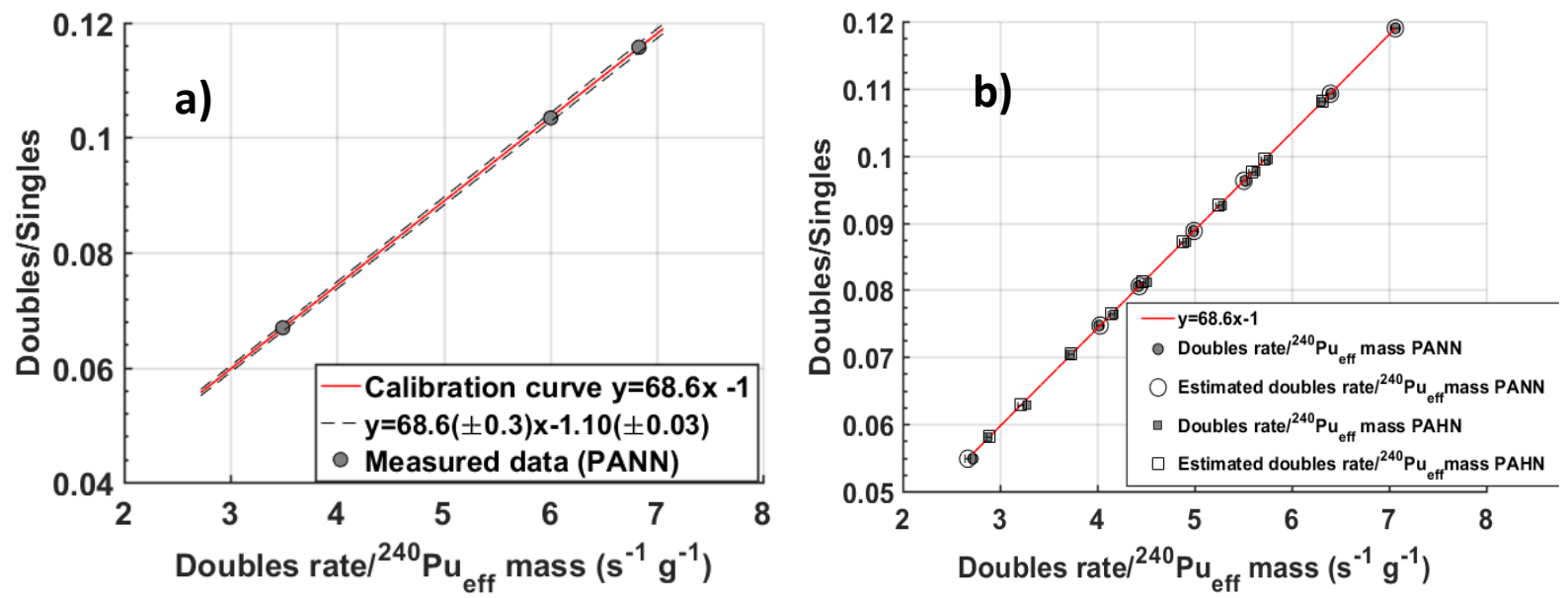
310 We used simulated neutron singles when measured data were not available, e.g., in the case of highly 311 radioactive PAHN samples acquired in coincidence mode. The combined standard uncertainty 312 associated with the ${ }^{240} \mathrm{Pu}_{\text {eff }}$ mass estimated with this method was comparable to the one achieved by 313 the single-parameter calibration method (Table 5). The absolute bias was negligible for test samples of 314 the same type of the calibration test series, i.e. PANN plates, while in general higher, in absolute value, 315 than the bias error of the single-parameter calibration procedure.

Table 5. Estimated ${ }^{240} \mathrm{Pu}_{\text {eff }}$ mass using the two-parameter calibration method.

\begin{tabular}{|c|c|c|c|c|c|c|c|c|c|c|}
\hline \multicolumn{11}{|c|}{ PAHN } \\
\hline Number of plates & 1 & 3 & 5 & 7 & 9 & 11 & 13 & 15 & 17 & 19 \\
\hline $\begin{array}{l}\text { Actual }{ }^{240} \mathrm{Pu} \\
\text { effective mass (g) }\end{array}$ & 25.05 & 75.14 & 125.23 & 175.32 & 225.42 & 275.51 & 325.60 & 375.69 & 425.79 & 475.88 \\
\hline Estimated ${ }^{240} \mathrm{Pu}$ & 24.90 & 76.66 & 124.96 & 176.34 & 227.75 & 277.66 & 327.65 & 378.49 & 428.98 & 474.73 \\
\hline effective mass (g) & \pm 0.35 & \pm 0.21 & \pm 0.19 & \pm 0.17 & \pm 0.16 & \pm 0.14 & \pm 0.13 & \pm 0.04 & \pm 0.04 & \pm 0.04 \\
\hline Absolute bias (g) & -0.15 & 1.52 & -0.27 & 1.02 & 2.33 & 2.15 & 2.05 & 2.80 & 3.20 & -1.15 \\
\hline \multicolumn{11}{|c|}{ PANN } \\
\hline Number of plates & 1 & 3 & 5 & 7 & 9 & 11 & 13 & 15 & 17 & 19 \\
\hline $\begin{array}{l}\text { Actual }{ }^{240} \mathrm{Pu} \\
\text { effective mass (g) }\end{array}$ & 4.70 & & 23.50 & 32.90 & 42.31 & 51.71 & & 70.51 & & 89.31 \\
\hline Estimated ${ }^{240} \mathrm{Pu}$ & 4.70 & n. a. & 23.50 & 32.90 & 42.31 & 51.71 & n. a. & 70.51 & n. a. & 89.31 \\
\hline effective mass (g) & \pm 0.34 & & \pm 0.21 & \pm 0.19 & \pm 0.17 & \pm 0.16 & & \pm 0.14 & & \pm 0.13 \\
\hline Absolute bias (g) & $<0.001$ & & $<0.001$ & $<0.001$ & $<0.001$ & $<0.001$ & & $<0.001$ & & $<0.001$ \\
\hline
\end{tabular}

317

318

319

320

321

322

323

324

325

326

327

328

329

330

331

332

333

\section{Summary and conclusions}

We designed and developed a fast-neutron multiplicity counter based on 8 EJ-309 liquid and 8 stilbene crystal detectors. The system geometry was optimized for the passive assay of plutonium metal samples of different composition. Two types of plutonium metal plates, with different ${ }^{240} \mathrm{Pu}$ effective mass, were used at Idaho National Laboratory: PAHN and PANN $\left(4.7 \mathrm{~g}\right.$ and $25.05 \mathrm{~g}{ }^{240} \mathrm{Pu}_{\text {eff }}$ mass per plate, respectively). The ${ }^{239} \mathrm{Pu}$ mass percentage was $74 \%$ for the PAHN and $95 \%$ for the PANN series. We measured neutrons emitted in coincidence by several plate assemblies, having an overall ${ }^{240} \mathrm{Pu}$ effective mass from $4.7 \mathrm{~g}$ to $476 \mathrm{~g}$.

Two calibration-based approaches were used to estimate the ${ }^{240} \mathrm{Pu}_{\text {eff }}$ mass of the samples. Both methods succeeded in estimating the ${ }^{240} \mathrm{Pu}_{\text {eff }}$ mass of an unknown sample with an uncertainty lower than $1 \%$ in a 4-minute assay time $\left({ }^{240} \mathrm{Pu}_{\text {eff }}>50 \mathrm{~g}\right)$. The statistical uncertainty is lower than the one achieved by standard ${ }^{3} \mathrm{He}$ counters for plutonium metal samples of similar mass. The difference between the estimated and the declared ${ }^{240} \mathrm{Pu}_{\text {eff }}$ mass, i.e., the bias error, measured by the fast-neutron multiplicity counter was consistently lower compared to the bias error of a standard thermal system. 
Further work is needed to assess potential limitations of the systems, such as operational drifts due to external temperature caused by the temperature dependence of the gain of photomultiplier tubes. In this work, spurious coincidences due to cross-talk were minimized by rejecting counts from nearest neighbor detectors, however new algorithms are being developed which are able to account for those events in the general framework of the multiplicity analysis equations. A promising property of the system is its position sensitivity, which will be further investigated and could be used to improve the characterization of unknown samples by measuring the known angular distribution of neutrons emitted by ${ }^{240} \mathrm{Pu}$ spontaneous fission [23].

\section{Acknowledgements}

The authors thank the staff at the ZPPR facility of Idaho National Laboratory for their assistance during the measurement campaign. This work is funded in-part by the Consortium for Verification Technology under Department of Energy National Nuclear Security Administration award number DE-NA0002534.

\section{References}

[1] M.S. Krick, J.E. Swansen, Neutron multiplicity and multiplication measurements, Nucl. Instr. Meth. Phys. Res. 219 (1984) 384-393.

[2] R.T. Kouzes, J.H. Ely, A.T. Lintereur, E.K. MacE, D.L. Stephens, M.L. Woodring, Neutron detection gamma ray sensitivity criteria, Nucl. Instr. Meth. Phys. Res. A. 654 (2011) 412-416.

[3] S.M. Robinson, R.C. Runkle, R.J. Newby, A comparison of performance between organic scintillation crystals and moderated $3 \mathrm{He}$-based detectors for fission neutron detection, Nucl. Instr. Meth. Phys. Res. A. 652 (2011) 404-407.

[4] H. Menlove, J. Stewart, S. Qiao, T. Wenz, P. Verrecchia, Neutron Collar Calibration and Evaluation for Assay of LWR Fuel Assemblies Containing Burnable Neutron Absorbers, Los Alamos National Laboratory report LA-11965-MS, 1990.

[5] G.F. Knoll, Radiation Detection and Measurement, 4th ed., John Wiley \& Sons, Hoboken, 2010.

[6] D. Henzlova, R. Kouzes, R. McElroy, P. Peerani, K. Baird, A. Bakel, M. Borella, M. Bourne, L. Bourva, F. Cave, R. Chandra, D. Chernikova, S. Croft, G. Dermody, A. Dougan, J. Ely, E. Fanchini, P. Finocchiaro, V. Gavron, M. Kureta, K.D. lanakiev, K. Ishiyama, T. Lee, C. Martin, K. McKinny, H.O. Menlove, C. Orton, A. Pappalardo, B. Pedersen, R. Plenteda, S. Pozzi, M. Schear, M. Seya, E. Siciliano, S. Stave, L. Sun, M.T. Swinhoe, H. Tagziria, J. Takamine, A.-L. Weber, T. Yamaguchi, H. Zhu, Current Status of Helium-3 Alternative Technologies for Nuclear Safeguards, Los Alamos National Laboratory report LA-UR-15-21201, 2015.

[7] D.L. Chichester, S.J. Thompson, M.T. Kinlaw, J.T. Johnson, J.L. Dolan, M. Flaska, S.A. Pozzi, Statistical estimation of the performance of a fast-neutron multiplicity system for nuclear material accountancy, Nucl. Instr. Meth. Phys. Res. A. 784 (2015) 448-454.

[8] E. Technology, NEUTRON/GAMMA PSD LIQUID SCINTILLATOR EJ-301, EJ-309, (n.d.). http://www.eljentechnology.com/images/products/data_sheets/EJ-301_EJ-309.pdf (accessed 
May 12, 2016).

373

374

375

376

377

378

379

380

381

382

383

384

385

386

387

388

389

390

391

392

393

394

395

396

397

398

399

400

401

402

403

404

405

[9] N. Zaitseva, A. Glenn, L. Carman, H. Paul Martinez, R. Hatarik, H. Klapper, S. Payne, Scintillation properties of solution-grown trans-stilbene single crystals, Nucl. Instr. Meth. Phys. Res. A. 789 (2015) 8-15.

[10] N.Z. Galunov, O.A. Tarasenko, V.A. Tarasov, Determination of the light yield of organic scintillators, Funct. Mater. 20 (2013) 304-309.

[11] CAEN S.p.A., V1730 user manual, (2016).

[12] J.K. Polack, M. Flaska, A. Enqvist, C.S. Sosa, C.C. Lawrence, S.A. Pozzi, An algorithm for chargeintegration, pulse-shape discrimination and estimation of neutron/photon misclassification in organic scintillators, Nucl. Instr. Meth. Phys. Res. A. 795 (2015) 253-267.

[13] J.B. Briks, The Theory and Practice of Scintillation Counting, 1st ed., Pergamon, Oxford, 1964.

[14] D. Reilly, N. Ensslin, H. Smith, eds., Passive Nondestructive Assay Manual, 1st ed., Los Alamos National Laboratory, Los Alamos, 1991.

[15] CAEN, GD2827 How to make coincidences with CAEN Digitizers, 2016.

[16] T.H. Shin, M.J. Marcath, A. Di Fulvio, S.D. Clarke, S.A. Pozzi, Neutron cross-talk characterization of liquid organic scintillators, in: 2015 IEEE Nucl. Sci. Symp. Med. Imaging Conf. NSS/MIC 2015, 2016.

[17] M.A. Norsworthy, A. Poitrasson-Rivière, M.L. Ruch, S.D. Clarke, S.A. Pozzi, Evaluation of neutron light output response functions in EJ-309 organic scintillators, Nucl. Instr. Meth. Phys. Res. A. 842 (2016) 20-27.

[18] D.L. Chichester, Properties of Nuclear Fuel Used in Tests with the LLNL Gamma-Ray Mirror in September 2014, Idaho National Laboratory report MIS-14-33068, 2014.

[19] C.W. Solbrig, J. Andrus, C. Pope, ZPPR Fuel Element Thermal Stress-Strain Analysis, World J. Nucl. Sci. Technol. 4 (2014) 123-128.

[20] J.L. Dolan, M. Flaska, A. Poitrasson-Riviere, A. Enqvist, P. Peerani, D.L. Chichester, S.A. Pozzi, Plutonium measurements with a fast-neutron multiplicity counter for nuclear safeguards applications, Nucl. Instr. Meth. Phys. Res. A. 763 (2014) 565-574.

[21] G. Cowan, Statistical Data Analysis, 1st ed., Clarendon Press, Oxford, 1998.

[22] S.A. Pozzi, S.D. Clarke, W.J. Walsh, E.C. Miller, J.L. Dolan, M. Flaska, B.M. Wieger, A. Enqvist, E. Padovani, J.K. Mattingly, D.L. Chichester, P. Peerani, MCNPX-PoliMi for nuclear nonproliferation applications, Nucl. Instr. Meth. Phys. Res. A. 694 (2012) 119-125.

[23] M.J. Marcath, T.H. Shin, S.D. Clarke, P. Peerani, S.A. Pozzi, Neutron angular distribution in plutonium-240 spontaneous fission, Nucl. Instr. Meth. Phys. Res. A. 830 (2016) 163-169. 